\title{
In Situ Characterization of the Self-assembly of a Polystyrene- Polydimethylsiloxane Block Copolymer during Solvent Vapor Annealing
}

\author{
W. Bai ${ }^{* 1}$, K. G. Yager ${ }^{* 2}$, C. A. Ross ${ }^{1, \#}$ \\ "Equal contributions \\ ${ }^{1}$ Dept. Materials Science and Engineering, Massachusetts Institute of Technology, \\ Cambridge MA 02139 \\ ${ }^{2}$ Center for Functional Nanomaterials, Brookhaven National Laboratory, Upton, NY \\ 11973 \\ "caross@mit.edu
}

\section{Supporting Materials}

\section{In Situ GISAXS Set-Up}

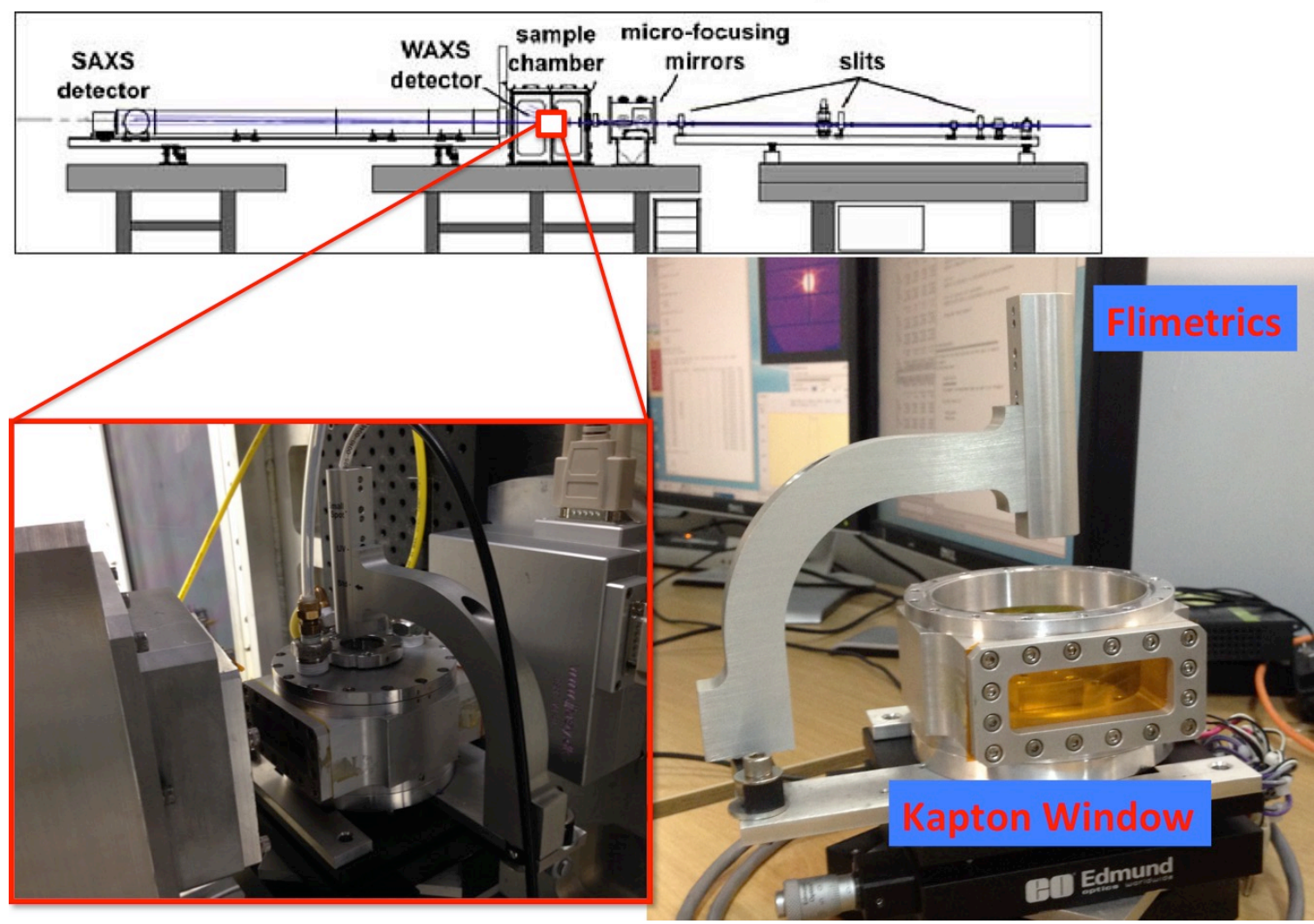

Figure S1. In Situ GISAXS experimental set-up for block copolymer solvent vapor annealing. 


\section{Section S1. GISAXS data analysis}

Grazing-incidence small-angle x-ray scattering (GISAXS) data were analyzed to obtain the lattice constants of the BCP hexagonal unit-cell, as well as the average grain size, and an estimate of the relative amounts of cylinder grains oriented in-plane vs. out-of-plane. GISAXS detector images were converted into reciprocal-space ( $q$-space) using a standard sample with scattering rings at known $q$ (silver behenate). To determine lattice constants, linecuts through the two-dimensional GISAXS images were taken. For the in-plane periodicity of the out-of-plane (vertically-oriented) cylinder population, a horizontal cut along the Yoneda, and through the $1^{\text {st }}$ GISAXS peak, was used (the intensity along the Yoneda improves the signal-to-noise of this determination). The peak was fit using a Gaussian with a power-law background (to account for, e.g. diffuse scattering), in order to obtain the peak center position $\left(q_{x}\right)$ and peak width $(\Delta q)$. This peak arises from the outof-plane (vertically-oriented) cylinder population, and its $q$-value can be used to compute the cylinder layering distance $L_{x}=2 \pi / q_{x}$. The corresponding cylinder-cylinder distance can be computed, for a hexagonal unit-cell, as $P_{x}=L_{x} \sqrt{3} / 2$. For the in-plane cylinder population (horizontal orientation), the $1^{\text {st }}$ peak above the Yoneda was used. This peak appears as a doublet in GISAXS, owing to the multiple possible scattering events, as described within the distorted-wave Born approximation (DWBA) framework. ${ }^{1,2} \mathrm{We}$ analyzed the peak centered about the direct-beam (rather than the reflected beam); i.e. the 'transmission channel'. ${ }^{3}$ A horizontal linecut was used to determine the $q_{x}$ position of this peak, which gives the in-plane periodicity $H_{x}$ of this in-plane (horizontal cylinder) population. To determine the out-of-plane (film normal direction) spacing of this population, the $q_{z}$ center of this same peak is obtained via a fit to a Gaussian function with a background. In this case, the peak position is corrected to account for the refraction-induced distortion to reciprocal-space, using the known incidence-angle and an estimate of the x-ray refractive index of the film (at $14.1 \mathrm{keV}$ ). The equations for this correction are well-known, ${ }^{1,3-7}$ and ultimately result from the application of Snell's law to the grazing-incidence geometry. Note that solvent uptake by the film would be expected to modify the refractive index; however, this change is subtle since the refractive index of the polymer film and of a bulk organic solvent are similar (especially compared to the $n=$ 1.0 refractive index of the ambient environment). The (corrected) $q_{z}$ component of this 'diagonal' peak is exactly half the $q_{z}$ value of the purely out-of-plane peak (which is hidden behind the beamstop/specular-rod). Thus, we compute the cylinder layering distance along the film-normal as $H_{z}=2 \pi /\left(2 q_{z}\right)$.

The average in-plane grain sizes were estimated by extracting the correlation-length using a Scherrer peak-width analysis applied to the $q_{x}$ linecut. ${ }^{8}$ Heuristically, broad peaks correspond to short distances over which positional order is maintained in the hexagonal cylinder lattice, whereas sharp peaks indicate large distances. The Scherrer analysis was performed taking into account the peak-broadening contributions from instrumental effects (wavelength spread of incident radiation, divergence of microfocused beam, detector pixel size), as well as the geometric broadening resulting from the grazingincidence geometry. ${ }^{9}$ 
The amounts of cylinders oriented in-plane vs. out-of-plane was estimated using the intensity of the corresponding scattering peaks. For each image, the full integrated intensity of the aforementioned peaks was computed, after the corresponding local background was subtracted. The $q_{x}$ peak along the Yoneda arises purely from the out-ofplane population, whereas the peak above the Yoneda arises from the in-plane population. The scattering intensity of a peak is linearly proportional to the amount of material that contributes to it (strictly, proportional to the scattering volume; i.e. the intersection between the beam and the given sub-population). We assume that our samples resemble in-plane powders (no preferred in-plane direction, confirmed by SEM images). Owing to the intersection of the Ewald sphere with reciprocal-space, the scattering peaks we observe arise from rings in the full three-dimensional reciprocal-space. This applies to both peaks of interest; thus, to a first approximation, no correction of intensities is necessary. The relative integrated intensities of the scattering peaks can be used as an estimate of the relative amounts of the two populations. We note that a $\sin (\chi)$ factor, where $\chi$ is the angle with respect to the $q_{z}$ axis, can be applied to account for the slightly different reciprocal-volume each peak represents. ${ }^{10,11}$ This suggests a relative correctionfactor (for out-of-plane population relative to in-plane) of $\sin \left(60^{\circ}\right) / \sin \left(90^{\circ}\right) \approx 0.9$. Conversely, the $q_{x}$ peak (vertical cylinders) arises from the in-plane averaging of 6 diffraction peaks, while the $q_{z}$ peak (horizontal cylinders) arises from the in-plane averaging of 2 diffraction peaks, imposing a correction factor of $6 / 4=1.5$. These two effects tend to offset one another: the combined correction is $\sim 1.3$. Finally, we note that the grazing-incidence intensity enhancement may not be strictly identical for the two peaks of interest. Despite performing a local background subtraction for each peak (and thus removing the baseline intensity of the Yoneda band), the $q_{x}$ peak may have a somewhat higher intensity enhancement, owing to its lower exit angle (this would impose a correction $<1.0$ ). Thus, while we interpret the relative scattering intensities as fractions of the two corresponding cylinder populations, we acknowledge a small systematic bias in the reported values. As such, we only attempt to interpret these values in a relative sense, in order to establish trends, and do not claim that the absolute population fractions are known unambiguously.

\section{Section S2. The Swelling Behavior of PS-PDMS in vapor from a Toluene: Heptane 5:1 volumetric liquid mixture}

In this work, $2500 \mathrm{~cm}^{3}$ toluene: heptane 5:1 volumetric liquid mixture was used in the bubbler of the solvent flow system. A decrease of liquid level in the bubbler was not found in the 5-hour annealing test, which indicated that the amount of solvent that was evaporated from the bubbler was small and the composition of the vapor therefore remained constant. The mole fractions of toluene and heptane in the vapor phase generated from a Toluene: Heptane 5:1 volumetric solvent mixture are: ${ }^{12}$

$$
\begin{gathered}
y_{\text {tol }}=\frac{p_{\text {tol }}^{*}}{p_{\text {total }}} \\
y_{\text {hep }}=\frac{p_{\text {hep }}^{*}}{p_{\text {total }}}
\end{gathered}
$$


where $p_{\text {tol }}^{*}$ and $p_{\text {hep }}^{*}$ are partial vapor pressures of tolune and heptane, and $p_{\text {total }}=$ $p_{\text {tol }}^{*}+p_{\text {hep }}^{*}$.

Based on Raoult's Law for non-ideal mixing, the partial pressures of toluene and heptane are:

$$
\begin{aligned}
p_{\text {tol }}^{*} \phi_{\text {tol }} & =p_{\text {tol }} \gamma_{\text {tol }} x_{\text {tol }} \\
p_{\text {hep }}^{*} \phi_{\text {hep }} & =p_{\text {hep }} \gamma_{\text {hep }} x_{\text {hep }}
\end{aligned}
$$

where $\phi_{t o l}$ and $\phi_{h e p}$ are fugacity coefficients of toluene and heptane; $\gamma_{t o l}$ and $\gamma_{h e p}$ are activity coefficients, and $x_{t o l}$ and $x_{h e p}$ are the mole fractions in the liquid solution. $p_{t o l}$ and $p_{\text {hep }}$ are the vapor pressures of pure solvents.

For the situation of heptane diluted in toluene, $\gamma_{\text {hep }} \sim 1.4$ and $\gamma_{t o l} \sim 1,{ }^{13}$ and assuming $\phi_{t o l}$ and $\phi_{\text {hep }}$ are equal, ${ }^{14}$ a 5:1 volumetric ratio of toluene and heptane liquid mixture approximately corresponds to a mole ratio of 6.89: 1 toluene: heptane.

Therefore,

$$
y_{\text {tol }}=\frac{p_{\text {tol }}^{*}}{p_{\text {total }}} \sim 0.73
$$

This fraction of toluene in the vapor is close to the regime found in prior work where PS and PDMS homopolymers have equal swelling ratio for mixtures of saturated total vapor pressure, as shown in figure $\mathrm{S} 2 \mathrm{a}$ which is reproduced here for convenience. ${ }^{12}$

In a computational approach based on the effective volume fraction in the $\mathrm{BCP}$ as a function of solvent vapor composition, ${ }^{15}$ we found that a toluene mole fraction in the vapor of around 0.68 gave an effective volume fraction of PDMS equal to that in the bulk $\mathrm{BCP}(f=0.33)$, as shown in figure $\mathrm{S} 2 \mathrm{~b}$, reproduced here for convenience.

Based on these results, we see that the vapor generated from a toluene: heptane 5:1 volumetric mixture, which has a toluene mole fraction in the vapor of $\sim 0.7$, swells both blocks equally so that the effective volume fraction $f$ in the swelled state is the same as the dry volume fraction. 
(a)
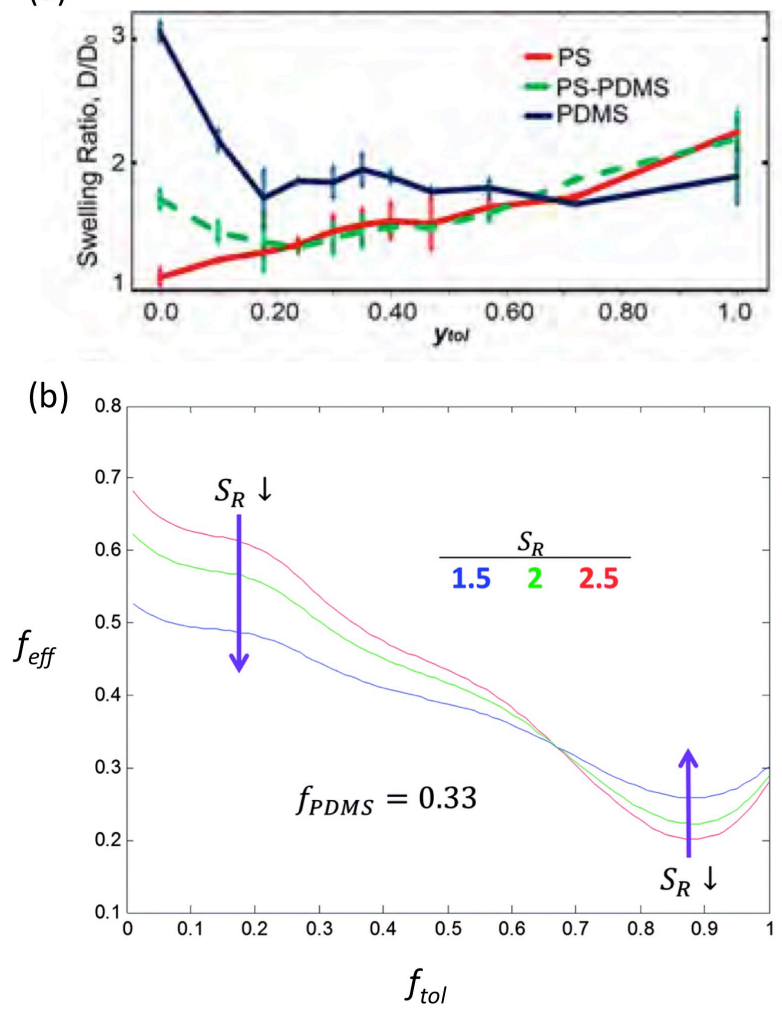

Figure S2. (a) Swelling ratios of PS, PS-PDMS, and PDMS for different mole fraction of toluene in a toluene and heptane vapor mixture. The plot is reproduced from [12]; (b) Plots of $f_{\text {eff }}$ using fitted selectivity parameters for toluene and heptane solvents in PSPDMS BCPs with given volume fraction of PDMS $f=0.33$ for three values of swelling ratio $S_{R}=1.5,2$, and 2.5. Plot is reproduced from [15]. 


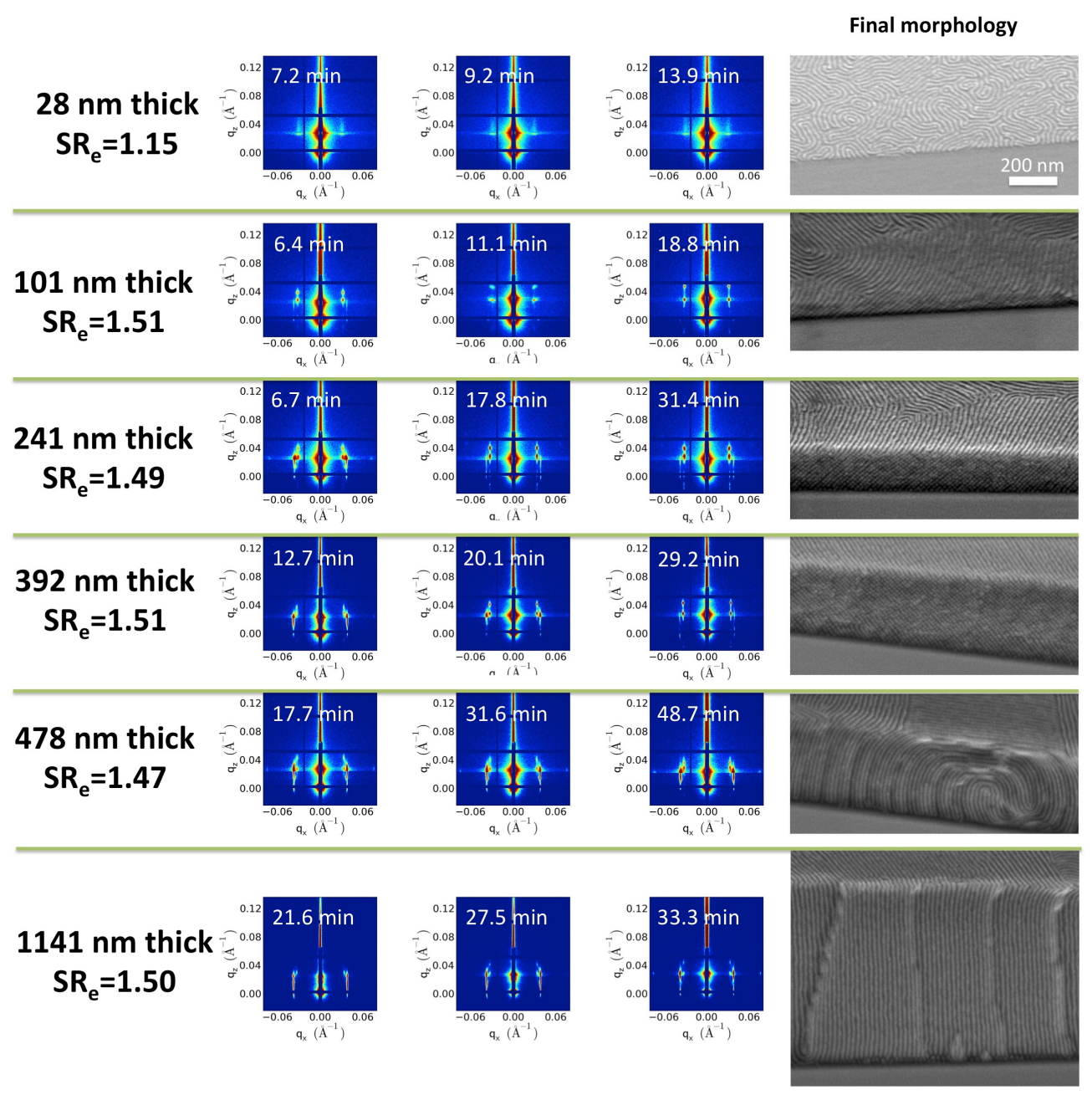

Figure S3. GISAXS at the solvent-BCP concentration equilibration stage, in PS- $b$-PDMS films with as-cast film thickness $28 \mathrm{~nm}, 101 \mathrm{~nm}, 241 \mathrm{~nm}, 392 \mathrm{~nm}, 478 \mathrm{~nm}, 1141 \mathrm{~nm}$, annealed under solvent vapor from a reservoir of Toluene: Heptane 5:1 volumetric mixture. The equilibrium swelling ratio was recorded for each sample, and the final etched morphology was imaged by SEM. 

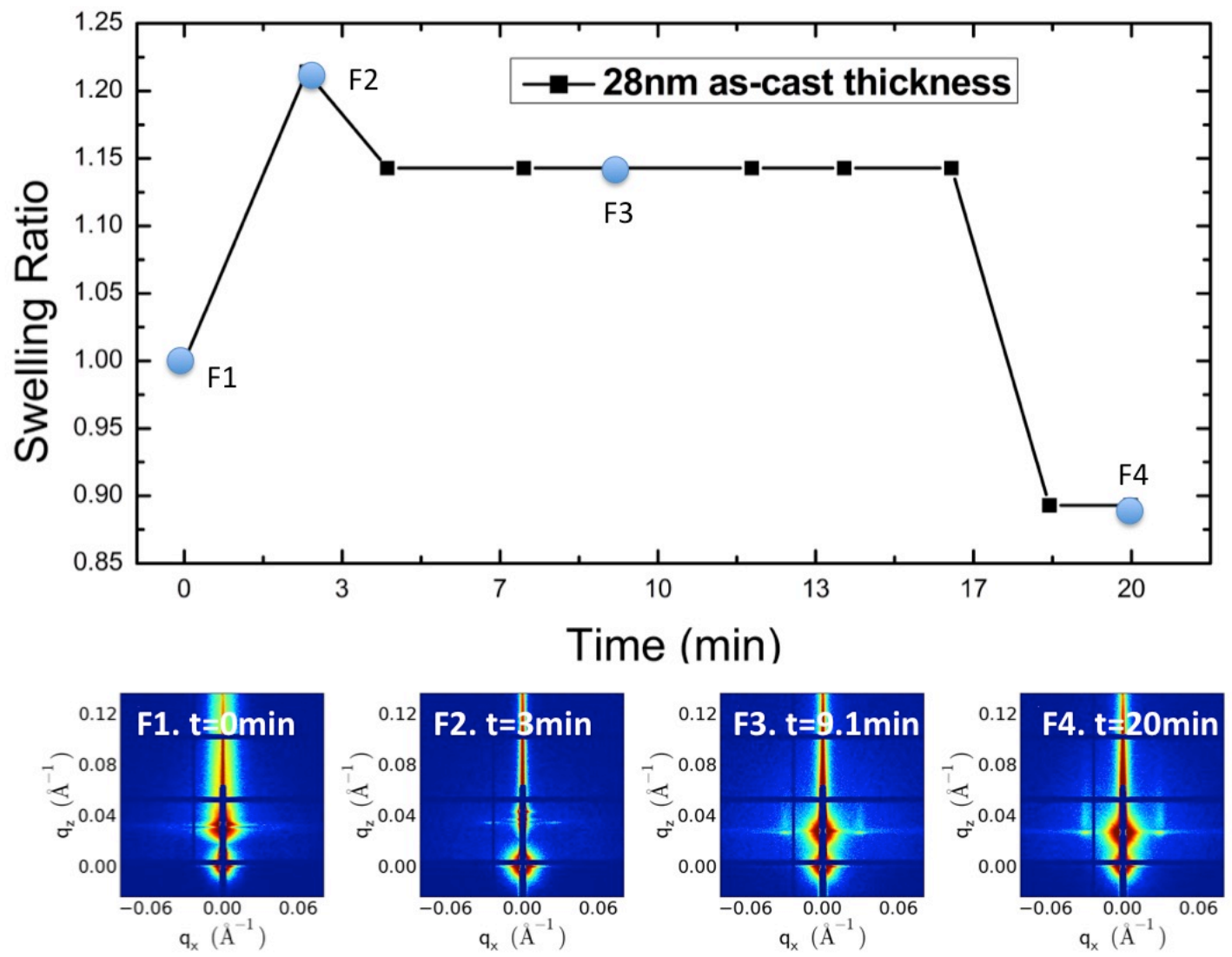

Figure S4. In Situ swelling ratio of SD16 PS- $b$-PDMS thin film with as-cast film thickness $28 \mathrm{~nm}$ (process F) under solvent vapor produced from Toluene:Heptane 5:1 volumetric mixture. F1, F2, F3, F4, in situ GISAXS measurements. 

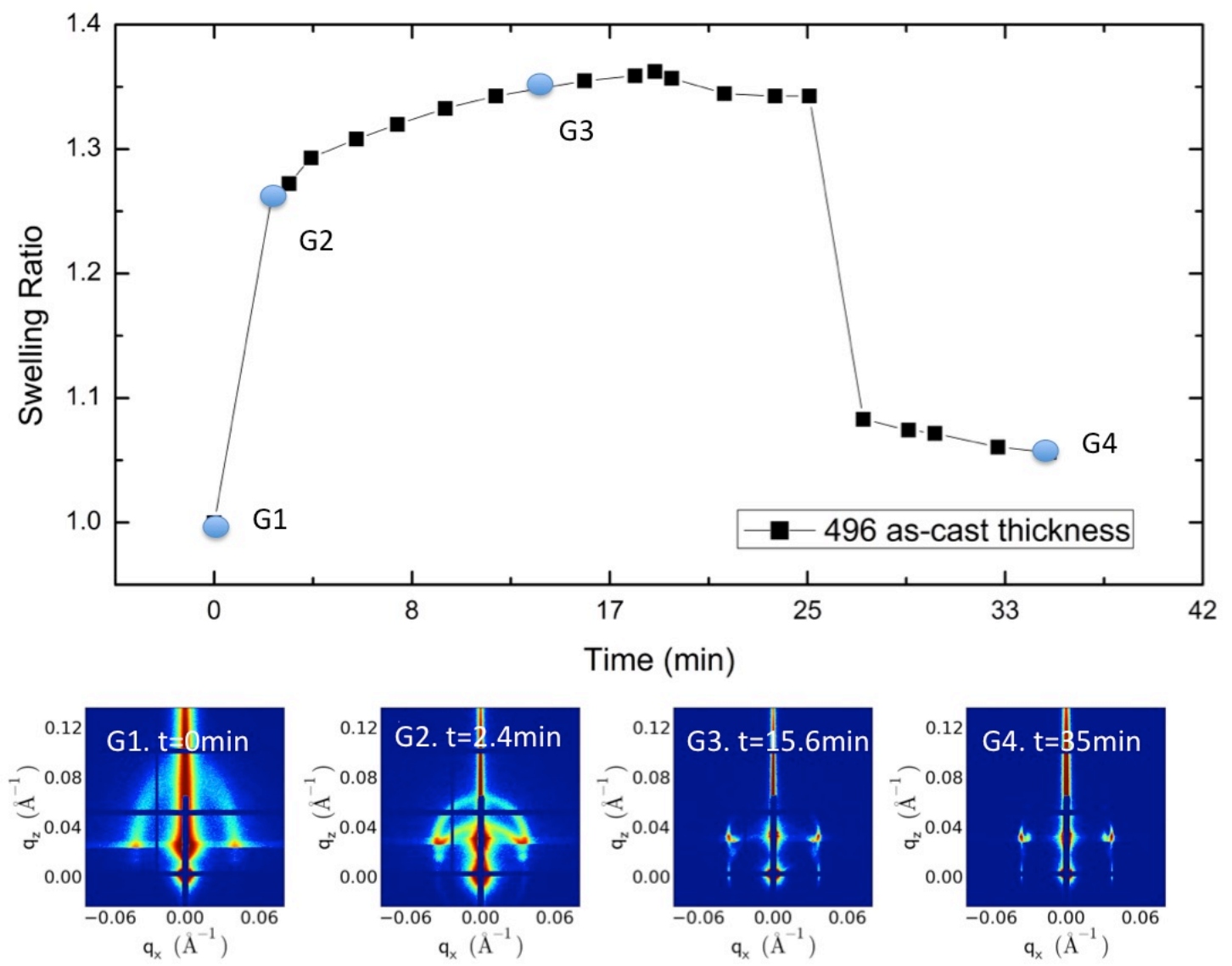

Figure S5. In situ swelling ratio of SD16 PS- $b$-PDMS thin film with as-cast film thickness $496 \mathrm{~nm}$ (process G) under solvent vapor produced from a Toluene:Heptane 5:1 volumetric mixture. G1, G2, G3, G4, show in situ GISAXS measurement data. 


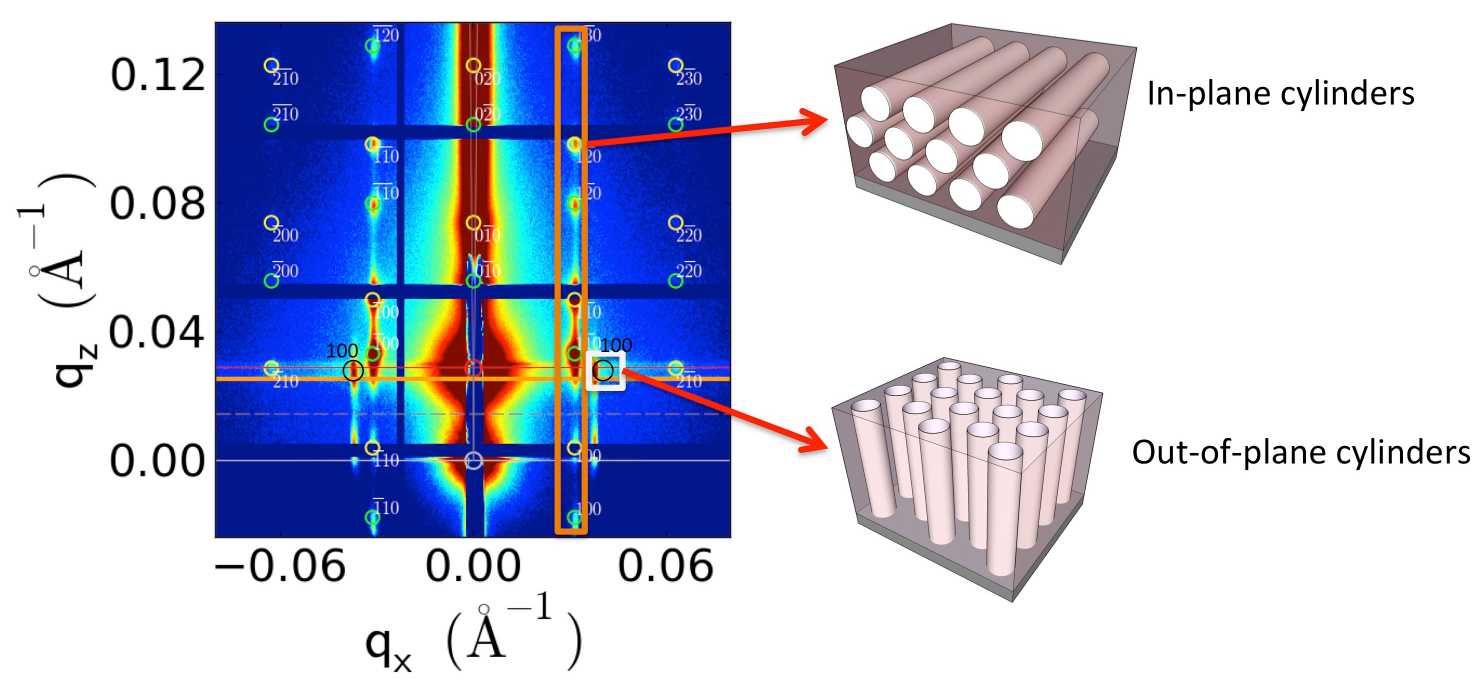

Figure S6. GISAXS of a solvent-annealed SD16 PS-PDMS block copolymer film with as-cast thickness $241 \mathrm{~nm}$. Two groups of scattering peaks coexisted in the GISAXS data, corresponding to in-plane cylinders and out-of-plane cylinders, respectively.

\section{References:}

[1] Lazzari, R.; Leroy, F.; Renaud, G. Phys. Rev. B 2007, 76, 125411.

[2] Renaud, G.; Lazzari, R.; Leroy, F. Surf. Sci. Rep. 2009, 64, 255-380.

[3] Lee, B.; Park, I.; Yoon, J.; Park, S.; Kim, J.; Kim, K.-W.; Chang, T.; Ree, M. Macromolecules 2005, 38, 4311-4323.

[4] Toney, M. F.; Brennan, S. Phys. Rev. B 1989, 39, 7963-7966.

[5] Lu, X.; Yager, K. G.; Johnston, D.; Black, C. T.; Ocko, B. M. J. Appl. Crystallogr. 2013, 46, 165-172.

[6] Busch, P.; Rauscher, M.; Smilgies, D.-M.; Posselt, D.; Papadakis, C. M. J. Appl. Crystallogr. 2006, 39, 433-442.

[7] Dag W. Breiby, Oliver Bunk, Jens W. Andreasen, Henrik T. Lemked, M. M. N. Simulating X-ray diffraction of textured films http://journals.iucr.org/j/issues/2008/02/00/ce5029/ (accessed Apr 23, 2015).

[8] Langford, J. I.; Wilson, A. J. C. J. Appl. Crystallogr. 1978, 11, 102-113. 
[9] Smilgies, D.-M. J. Appl. Crystallogr. 2009, 42, 1030-1034.

[10] Page, K. A.; Kusoglu, A.; Stafford, C. M.; Kim, S.; Kline, R. J.; Weber, A. Z. Nano Lett. 2014, 14, 2299-2304.

[11] Johnston, D. E.; Yager, K. G.; Hlaing, H.; Lu, X.; Ocko, B. M.; Black, C. T. ACS Nano 2014, 8, 243-249.

[12] Gotrik, K. W.; Hannon, A. F.; Son, J. G.; Keller, B.; Alexander-Katz, A.; Ross, C. A. ACS Nano 2012, 6, 8052-8059.

[13] Wauquier, J. P. Petroleum Refining. Editions OPHRYS, 2000.

[14] Matsoukas, T. Fundamentals of Chemical Engineering Thermodynamics: With Applications to Chemical Processes; Prentice Hall, 2013.

[15] Hannon, A. F.; Bai, W.; Alexander-Katz, A.; Ross, C. A. Soft Matter 2015, 11, 3794 